\title{
Cabazitaxel Regimen
}

National Cancer Institute

\section{Source}

National Cancer Institute. Cabazitaxel Regimen. NCI Thesaurus. Code C160797.

A regimen consisting of cabizitaxel that may be used in the treatment of metastatic, castration-resistant prostate cancer (mCRPC). 